\title{
MS122.P10
}

\section{Rhombohedral distortion analysis on Pd(111) films under hydrogen loading}

Takashi Harumoto $^{1}{ }^{\text {, Takashi Ishiguro }}{ }^{2}$, Ji Shi $^{1}$, Yoshio Nakamura ${ }^{1}$

${ }^{1}$ Department Of Materials Science And Engineering, Tokyo Institute Of Technology, Tokyo, Japan, ${ }^{2}$ Department of Materials Science and Technology, Tokyo University of Science, Tokyo, Japan E-mail: harumoto.t.aa@m.titech.ac.jp

The palladium (Pd) films are applied for hydrogen sensors and hydrogen purification processes. However, their performances would be degraded after the hydrogen loading-unloading cycles. This degradation derives mainly from the accumulation of defects introduced by the lattice expansion-shrinkage during the loading-unloading cycles. Thus, the analyses on the strain and stress in the films during the hydrogen loading are important. The diffraction stress analysis is a powerful method for it, however, the elastic constants of Pd-hydride are required. Since the constants exhibit the hydrogen composition dependence, it is required to measure the composition for considering the dependence or employ the approximation such as disregarding the composition dependence. The former introduces the experimental difficulty while the latter results in the decrease in accuracy.

The rhombohedral distortion analysis is the distortion analysis method for (111)-textured face-centred cubic (fcc) films and is based on the primitive rhombohedral cell of fcc. The results of this analysis are the angle and the lattice parameter of the rhombohedral cell. The angle is related to the in-plane stress while the lattice parameter corresponds mainly to the lattice expansion-shrinkage. The main advantage of the analysis is that only two (111) lattice spacings at tilt angles of $0^{\circ}$ and $70.5^{\circ}$ are required and no elastic constant is employed during the calculation. Thus, it is suitable especially for the cases such as the elastic constants are unknown or have some dependences.

In this study, the strain in the (111)-textured Pd films during the hydrogen loading-unloading was investigated using the rhombohedral distortion analysis. It was revealed that the films exhibit the selective lattice expansion along the substrate normal direction at the low hydrogen composition phase. The expansion originates from the large in-plane compressive stress. In contrast, although the compressive stress yet remains large, the selective lattice expansion was suppressed at the high hydrogen composition phase. This indicates that the plastic deformation occurred during the formation of the high hydrogen composition phase. Accordingly, the hydrogen composition dependences on the compressive stress and the plastic deformation were discussed.

[1] Harumoto, T. et al. (2014) J. Appl. Cryst. 47, 1490-1501.

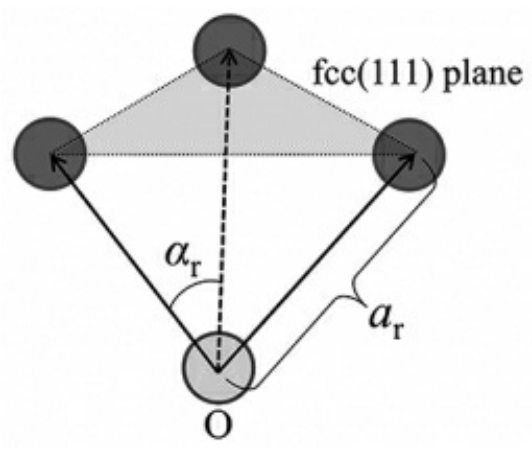

The angular change corresponds to the in-plane stress:

$\Delta \alpha_{\mathrm{r}}=\alpha_{\mathrm{r}}-60^{\circ} \approx \frac{4 \sqrt{3}}{9} \frac{1}{2} S_{2}^{111} \sigma_{\|}$

*Only the three basis vectors of the primitive rhombohedral cell of fcc are drawn.

Keywords: hydrogen, palladium, lattice strain 